\title{
Design, Manufacture and Test for Reliable 3D Printed Electronics Packaging
}

\author{
T. Tilford ${ }^{*}$, S. Stoyanov , J. Braun², J. C. Janhsen², M.Burgard², R. Birch³, and C. Bailey \\ [1] Computational Mechanics and Reliability Group, University of Greenwich, Park Row, Greenwich, London UK \\ [2] Fraunhofer Institute for Manufacturing Engineering and Automation IPA Nobelstr. 12, Stuttgart, Germany \\ [3] Microsemi Corporation, Castlegate Business Park, Caldicot, Monmouthshire, UK \\ Email: T.Tilford@gre.ac.uk
}

Abstract:

The development of a surrogate modelling approach to aid design of 3D printed electronics packaging structures is presented, alongside a detailed overview of manufacture and reliability of a representative test structure. An overview of the current status in 3D printing in the electronics packaging sector is provided. Subsequently, a surrogate modelling approach for correlating thermomechanical stresses within a package to a number of design parameters is presented. This approach enables the design of a package to be considered in a more insightful manner and can additionally be integrated into condition based monitoring tools capable of enhancing product robustness. An overview of an advanced electronics packaging system capable of 3D printing electronics packages is presented. The system combines inkjet printing and curing of multiple materials, including conductive silver inks, with precision component placement, multi-material dispensing and 3D inspection systems to provide a highly flexible solution for rapid manufacture of electronics packages. Test structures manufactured using the system were subjected to a vigorous set of reliability tests. Details of the test regime and related results are presented. All tests were passed, indicating the robustness of the described manufacturing process.

The key originality of the work is that it provides a comprehensive overview of the journey from design assessment an optimisation, through the manufacturing process and on to reliability testing. Areas of novelty in this work are associated with the development of fast, accurate surrogate models able to predict key reliability factors in response to a range of design parameters and insight into the development of a 3D manufacturing system for electronics packaging.

Keywords - 3D Printing; Electronics Packaging; Design Tools; Modelling; Reliability

\section{Introduction}

3D printing, and in general Additive Manufacturing, has received significant attention from many industries in the past few years, including form electronics manufacturers. This interest has led to the development and commercialization of a range of materials for use with different additive manufacturing techniques, design tools and software and 3D printers. These have been gradually adopted and have now started to be used for digital manufacturing of products or parts in many sectors such as aerospace, medical and construction.

The main advantages of 3D printing are that they are cost-effective, offer high throughput combined with mass-customization capability and are material and energy efficient. In addition they enable full digitalization of the whole manufacturing process [1]. While 3D printing is still considered primarily as very advantageous in prototyping, more recently these techniques have additionally started to be adopted in manufacturing lines.

$3 \mathrm{D}$ printing as an approach to electronics manufacture and packaging is now firmly on the agenda for many manufacturers in the sector. Examples of 3D printing in the electronics packaging sector include the fabrication of thin-film transistors, conductive and photovoltaic structures, mechanical actuators and sensors [2, 3]. Recent advances in the technology suggest that 3D printing has the potential to transform the traditional manufacture of electronic products into printing-based manufacture of completely integrated devices with functional capabilities [4]. 
The large number of academic research 3D printing systems targeting the electronics packaging sector $[5,6]$ are now augmented by a number of commercially available systems intended for production of saleable products. The Nano Dimension dragonfly [7] is a high resolution Multilayer PCB prototyping system featuring a build size 200mm square, 80-100 $\mu \mathrm{m}$ traces, $150 \mu \mathrm{m}$ interconnects, with a minimum layer thickness of $3 \mu \mathrm{m}$. These systems provide an extremely rapid prototyping capability for electronics manufacturers.

An example of a more flexible 3D printing system for the manufacture of electronics packaging would be the Optomec system [8] which is capable of forming packages with $30 \mu \mathrm{m}$ line width (+/-20\%), $50 \mu \mathrm{m}$ pad width, $60 \mu \mathrm{m}$ pitch between lines, line length of up to $1.5 \mathrm{~mm}$ and forming stacked die systems. The system achieves this printing resolution through combining Fused Deposition Modelling (FDM) and Aerosol Jet Additive manufacturing paradigms. The utilization of hybrid additive manufacturing systems offers a higher degree of flexibility in manufacturing and can be viewed as an important factor in facilitating effective manufacture of complex electronics packaging structures.

The EU project NextFactory [9] has developed a 3D printing, micro-deposition, micro-assembly, and curing system that will accurately deposit and cure both functional and structural materials and place/embed components in an integrated manner within a single platform. The system also uses a hybrid approach in order to increase its flexibility, with an inkjet system augmented by microdeposition tools that enable conductive adhesive materials to be used alongside silver nano-inks for conductive features. The system, illustrated in Figure 2, is modular in nature, formed of four physical modules and one virtual module. The three inkjet systems can print three separate materials (support/insulating/conducting), with four Fuji Dimatix inkjet printheads used for each material, an integrated LED UV light source for pinning and a flattening unit. The cure module is equipped with UV, IR and NIR systems. The assembly/dispense module is equipped with four grippers and 3 dispensing units, while the inspection module has 2D, 2.5D and 3D camera systems. An additional logistics unit with heated tray is incorporated into the system. The build area is $100 \times 100 \times 50 \mathrm{~mm}$ allowing multiple smaller components to be formed simultaneously. The min layer thickness is 10 $\mu \mathrm{m}$ with track width and pitch of 150 and $250 \mu \mathrm{m}$ respectively. A print time of $7 \mathrm{~s}$ per layer enables products to be formed in a rapid manner. In addition to the physical modules, illustrated in figure 3 , a virtual condition based monitoring module is incorporated into the system control software. This system utilises sensor and inspection system data in combination with machine learning algorithms and/or surrogate models to predict and optimize product quality during the manufacturing process. The condition based monitoring system is more fully explained in e.g. $[10,11]$.

While the use of a condition based monitoring approach can optimize the build quality of a 3D printed component, the reliability of the package will clearly be heavily influenced by its design. Furthermore, the robustness of a 3D printed package will need to be assessed through performing conventional reliability tests. Sections 2 and 3 of this paper discuss the application of a surrogate modelling approach to understand the influence of design parameters on package reliability, while section 4 discusses manufacture and test of a 3D printed test structure.

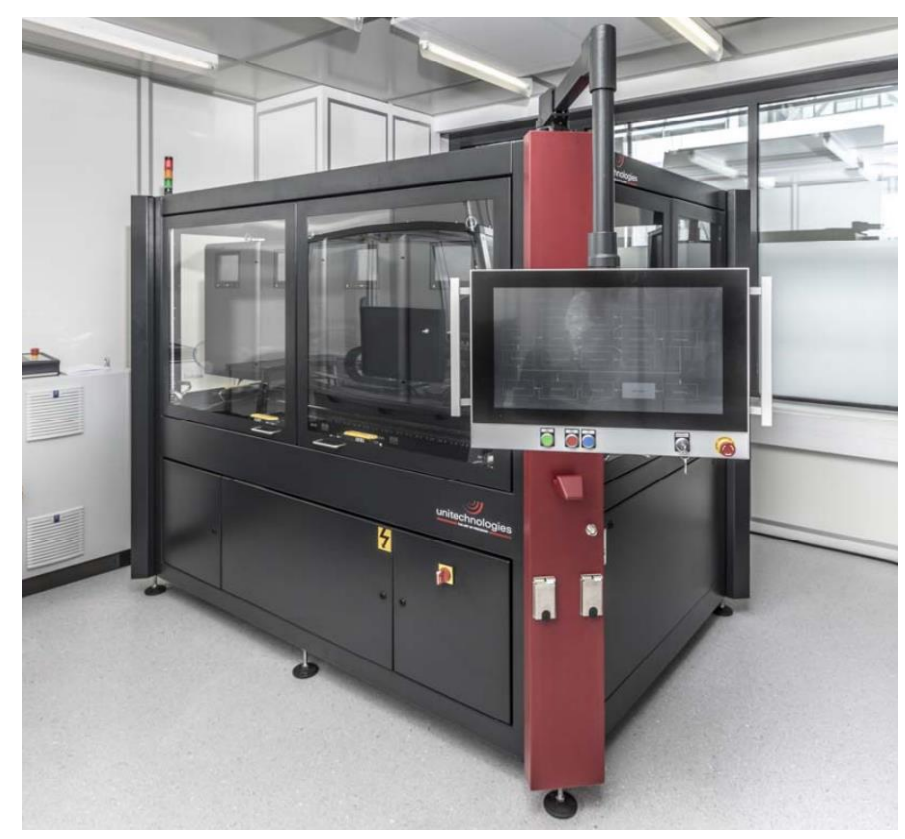

Figure 2: The NextFactory 3D manufacturing system 


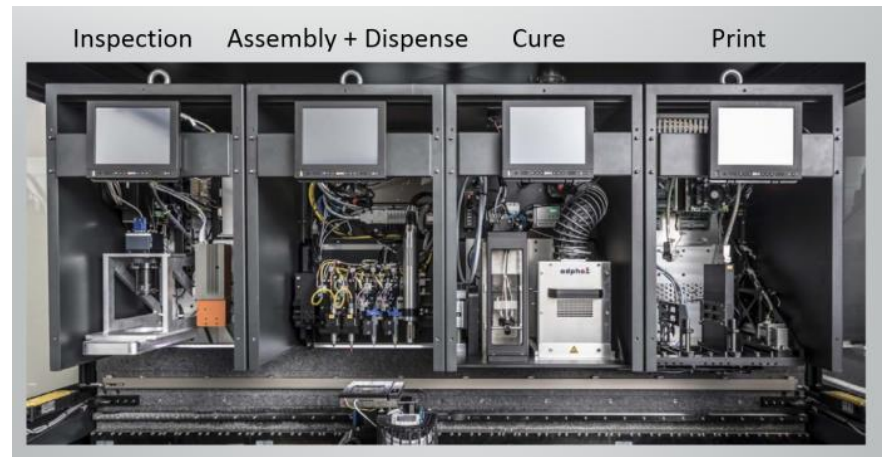

Figure 3: Interior view of the the NextFactory 3D manufacturing system showing modular nature of printing, cure, assembly, dispense and inspection

\section{Design for Reliability using Surrogate Modelling}

Advanced numerical techniques such as those based on finite elements can provide in-depth and valuable insights into various aspects of the process and its performance, as well as the quality and reliability of the printed parts, such analysis approaches are in general computationally expensive and require special user skills and expertise. The run time of such simulations often present a barrier for their adoption and not practical for design exploration, risk analysis and optimization. Adopting simple, and sometimes less accurate models, which are capable to provide fast predictions offers clear advantages in these types of analyses.

\subsection{Considerations for adopting surrogate models}

Derivation of surrogate models for 3D printing based on Design of Experiments (DoE) (and similarly Design of Simulations) and response surface (RS) modelling is considered to be an excellent approach that can support various DfX (Design for Excellence, a product development approach intended to enhance product quality) activities. Here we demonstrate the approach of surrogate modelling in constructing a predictive model for the stress in printed conductive lines as function of several design variables including a loading condition which can be used to identify the design of reliable printed parts. Understanding, through design space exploration and characterisation, how these parameters impact the magnitude of the induced thermo-mechanical stress due to coefficient of thermal expansion (CTE) differential miss-match between the substrate and the sintered silver traces can help designers to minimise the risks of delamination damage and unacceptable level of warpage and thus design products with improved reliability performance.

The geometry assessed in this study was developed as part of a more comprehensive analysis of the capabilities and limitations of 3D printing in the electronics packaging sector. The geometry defined consists of an insulating layer formed from an inkjet printed acrylic material with a series of tracks, formed from a silver nanomaterial ink, printed on the upper surface. A number of the tracks bridge embedded zero Ohm resistors. This study did not consider vias or multiple layers in this study but have been assessed in a more advanced iteration. The study, also, does not aim to compare the performance of the 3D printed part with a conventional FR4 / copper part. This work has been performed and will be published in future.

The design consists of a number of straight tracks and a number of meandering tracks. The intent of the design was to assess the bounds of the manufacturing process in terms of track and gap. The initial expectation was that the broad track/gap features could be readily printed but issues would arise regarding the finer track/gap features. The test structure dimensions are $25 \mathrm{~mm} \times 15 \mathrm{~mm} \times 0.5$ $\mathrm{mm}$.

Printing of physical prototypes showed that the process utilised was capable of printing the fine details and the design could have been more ambitious. A CAD model of the basic geometry is presented in Figure 4.

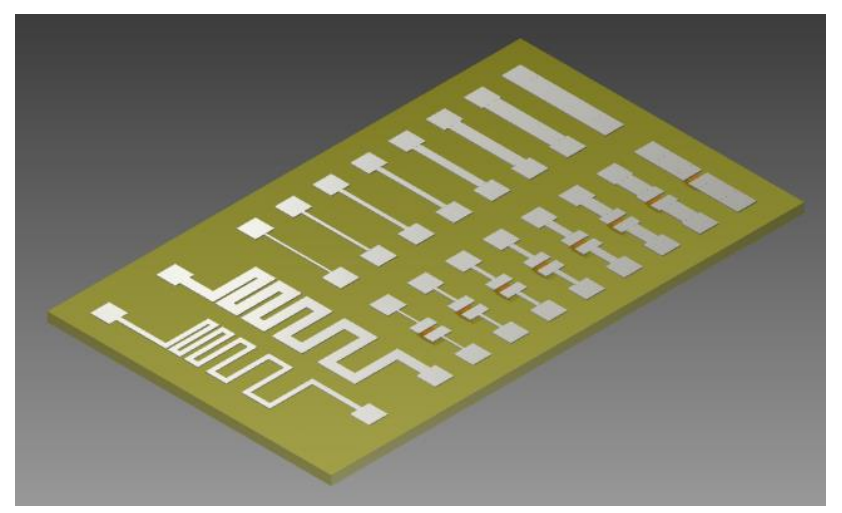

Figure 4: CAD model of test structure geometry 


\subsection{Surrogate Modelling for 3D printed Electronics}

In order to develop a simple model for predicting thermo-mechanical stresses in printed structures subjected to test, operational or environmental conditions (e.g. thermal cycling), we design and parameterize a configuration of printed traces on an insulating material. Such an approach will support the creation of design rules for minimizing the risk of delamination failure. Stress response of the printed structure to thermal load is evaluated using linear elastic finite element analysis. The design of the printed structure (in CAD format) and the finite element mesh of the model are shown in Figure 5. The printed structure is formed of a series of layers each approximately $9 \mu \mathrm{m}$ thin, printed at $400 \times 400 \mathrm{dpi}$. Cure shrinkage and thermal effects induce thermomechanical strains within the structure. These induce warpage and impact upon board reliability. It is, however, beyond the scope of this work to develop and utilize a model that captures the interaction between the very large number of layers in the board. As such, the board is considered to be formed from a single homogenous layer. Further work is required to investigate the magnitude of this approximation and to investigate the sensitivity of internal stresses to manufacturing process parameters.

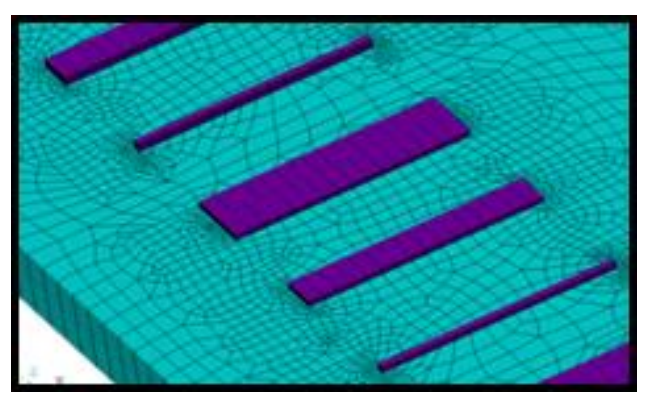

Figure 5: Finite element mesh utilised in this study.

The array of straight conductive lines is used to observe the stress change when width and height of the line varies. The length of each straight line in the pattern is $3.5 \mathrm{~mm}$. Width and thickness of a line are defined with values from the sets $(0.1 \mathrm{~mm}, 0.5 \mathrm{~mm}, 0.9 \mathrm{~mm})$ and $(0.05$ $\mathrm{mm}, 0.10 \mathrm{~mm}, 1.15 \mathrm{~mm}$ ) respectively. The nine lines in the above model represent all possible combinations of line width and line height as given with the two sets of values. The meander patterns are included in the model to inform stress responses in case of more complex geometrical design of the lines. Each meander is defined with width and height values that are taken as: (1) line width is either $0.250 \mathrm{~mm}$ or $0.500 \mathrm{~mm}$, and (2) line height is either $0.050 \mathrm{~mm}$ or $0.150 \mathrm{~mm}$. The ends of the lines contain square contact pads. Stress results for the snakelike curved patterns are not detailed in this paper and will be published separately.

The nominal design of the structure assumes thickness of the insulating material $1.0 \mathrm{~mm}$. The post-cure elastic modulus (E) and coefficient of thermal expansion (CTE) properties of the insulating ink (typically an acrylic) and the sintered conductive ink (conductive lines) are:

- Cured insulating ink: $\mathrm{E}=2.0 \mathrm{GPa}$ and $\mathrm{CTE}=65 \mathrm{ppm} /{ }^{\circ} \mathrm{C}$

- Sintered silver ink: $\mathrm{E}=7.0 \mathrm{GPa}$ and $\mathrm{CTE}=18 \mathrm{ppm} /{ }^{\circ} \mathrm{C}$

These values were not derived experimentally and are sourced from public domain sources following a literature review. Primary data is obtained from $\mathrm{Hu}$ [12] and Vasiljevic et al [13]. The properties of ink may be particularly sensitive to process parameters with further work required to provide more accurate estimates of these parameters.

Table I details the nominal values of design parameters that define the printed structure as well as design range for these (actual and normalised). The latter definitions are used in a later section of this paper detailing the derivation of a simple predictive surrogate model. 


\begin{tabular}{|c|c|c|c|c|}
\hline \multicolumn{3}{|c|}{ Parameter } & \multirow{2}{*}{$\begin{array}{c}\text { Parameter } \\
\text { Actual } \\
\text { Range } \\
\end{array}$} & \multirow{2}{*}{\begin{tabular}{|c} 
Parameter \\
Normalised \\
Range \\
\end{tabular}} \\
\hline $\begin{array}{c}\text { Notat } \\
\text { ion }\end{array}$ & Description & $\begin{array}{c}\text { Nominal } \\
\text { Value }\end{array}$ & & \\
\hline$\times 1$ & $\begin{array}{c}\text { Width of } \\
\text { conductive line } \\
(\mathrm{mm})\end{array}$ & 0.5 & $0.1-0.9$ & -1 to 1 \\
\hline $\mathrm{X} 2$ & $\begin{array}{l}\text { Thickness of } \\
\text { conductive line } \\
(\mathrm{mm})\end{array}$ & 0.10 & $0.05-0.15$ & -1 to 1 \\
\hline X3 & $\begin{array}{l}\text { Thickness of } \\
\text { insulating } \\
\text { material }(\mathrm{mm})\end{array}$ & 1.0 & $0.5-1.5$ & -1 to 1 \\
\hline$X 4$ & $\begin{array}{l}\text { Insulating } \\
\text { material CTE } \\
\left(\mathrm{ppm} /{ }^{\circ} \mathrm{C}\right)\end{array}$ & 65 & $30-100$ & -1 to 1 \\
\hline$\times 5$ & $\begin{array}{c}\text { Insulating } \\
\text { material } \\
\text { elastic } \\
\text { modulus (GPa) }\end{array}$ & 2.0 & $0.5-3.5$ & -1 to 1 \\
\hline X6 & $\begin{array}{c}\text { Applied } \\
\text { temperature } \\
\text { load, } \Delta \mathrm{T}\left({ }^{\circ} \mathrm{C}\right)\end{array}$ & 60 & $20-100$ & -1 to 1 \\
\hline
\end{tabular}

Table I: Printed electronics structure parameters and range of design parameters

The stress response of the structure is predicted using the developed finite element model under thermal load $\Delta \mathrm{T}=60^{\circ} \mathrm{C}$. This analysis implicitly accounts for the actual post-cure dimensions of the insulating ink and assumes no impact from potential residual stress in the peripheral region of the cured insulating material. The conductive ink is assumed to be photonic sintered and with no shrinkage taking place after the formation of the sintered silver domain exhibiting elastic behaviour. If the material behaviour is such that process temperature or/and cure shrinkage cause residual stress, then more detailed, inelastic modelling approach as formulated in section 2 of this paper will need to be adopted.

\subsection{FEA Stress Results for Nominal Structure}

Risks of thermo-mechanical damage, in particular in relation to delamination of printed conductive lines, depends on the level of interfacial stresses and the adhesion strength of the sintered conductive ink material. At present, there is no data available in this study that can be used to correlate FEA stress predictions with a corresponding damage stress level criterion. Therefore, presented analysis is predominantly qualitative: stress predictions can be used to inform the relative risk of damage and to support design decision that minimise the risk of failure.

The damage parameter used in this investigation is the maximum stress intensity calculated as an average element value in the location of highest stress. Stress intensity ( $\left.\sigma_{I N T}\right)$ is defined as:

$$
\sigma_{I N T}=\max \left(\left|\sigma_{1}-\sigma_{2}\right|,\left|\sigma_{2}-\sigma_{3}\right|,\left|\sigma_{3}-\sigma_{1}\right|\right)
$$

where $\sigma_{1}, \sigma_{2}, \sigma_{3}$ are the principal stress components. Figure 6 shows the predictions for stress intensity in the modelled structure in the case of the nominal design.

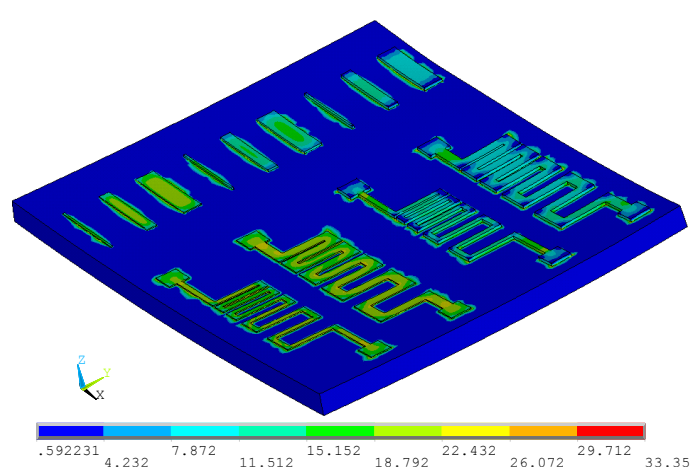

Figure 6: Contour plot of stress intensity $(\mathrm{MPa})$ in the printed structure with nominal design specification.

Deformed shape is magnified by factor $f=20$.

First, we observe the effect of the conductive line width on stress induced in the material under the nominal thermal load $\left(\Delta T=60^{\circ} \mathrm{C}\right)$ for the simulated inkjet-printed structure. Stress predictions are detailed in Figure 7. The contours of stress intensity are visualised at the lineinsulator interface (i.e. view at the "bottom side" of the conductive line). Larger width of the conductive line causes larger stress at the location of stress concentration. Peak stresses for line (tracks) with widths above $0.5 \mathrm{~mm}$ and thickness of $0.1 \mathrm{~mm}$ develop at the corners of the conductive lines. As the line width decreases, the maximum stress also decreases and at the same time shows tendency to shift the location of stress concentration in the region of the mid-side of the long edges. For lines with small width, the entire central region, 
half-way along the line length, is uniformly stressed. The maximum stress predicted in the case of the line with width $0.9 \mathrm{~mm}$ is $33 \%$ larger compared with the maximum stress predicted for the line with width $0.1 \mathrm{~mm}$.
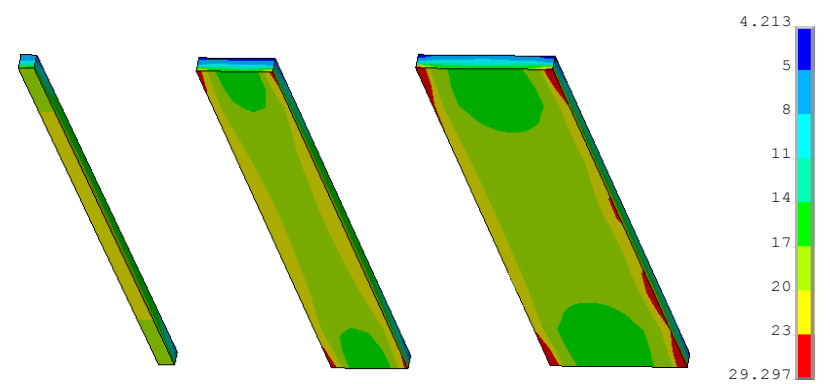

Figure 7: Contour levels of stress intensity for straight printed lines at interface with insulator in MPa. Width of lines is $0.1 \mathrm{~mm}, 0.5 \mathrm{~mm}$ and $0.9 \mathrm{~mm}$ and thickness of all three lines is $0.1 \mathrm{~mm}$.

Stress predictions for the straight lines show that thinner tracks are more compliant with the insulating material beneath. Stress distributions are also more uniform across the thinner lines, including at the interfaces with the insulator. Thicker lines show less uniformity in stress distribution, with stress peaking at the corners. Figure 8 shows the stress intensity distribution in conductive lines with varying line thickness. It can be concluded that the chance for interfacial crack initiation and propagation is more likely in the case of thick lines as the stress intensity level is higher compared with the case of thin lines. There is also a thickness level below which the stress concentration shifts from the line corners to the peripheral long edges of the line. The maximum stress value predicted in the case of the line with thickness 0.15 $\mathrm{mm}$ is $16 \%$ larger compared with the stress value for the line with thickness $0.05 \mathrm{~mm}$.
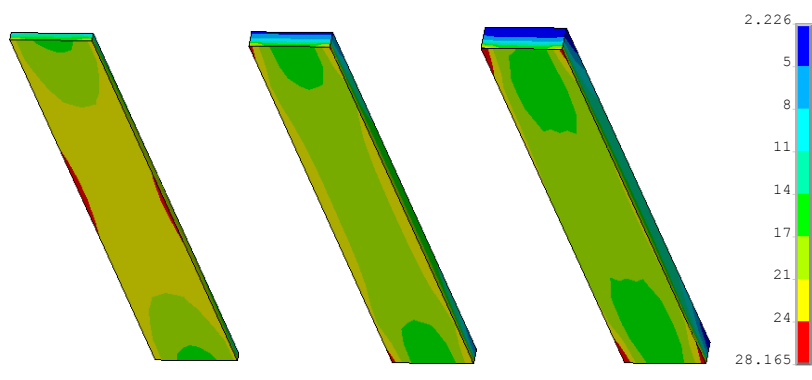

Figure 8: Contour levels of stress intensity for straight printed lines at interface with insulator in MPa. Thickness of lines is $0.05 \mathrm{~mm}, 0.1 \mathrm{~mm}$ and $0.15 \mathrm{~mm}$ (from left to right) and width of all three lines is $0.5 \mathrm{~mm}$.

The parametric study results on conductive line width and thickness, showing the effect of low and high range value for each of these parameters as detailed in Table 1, are summarised with the graph in Figure 9.

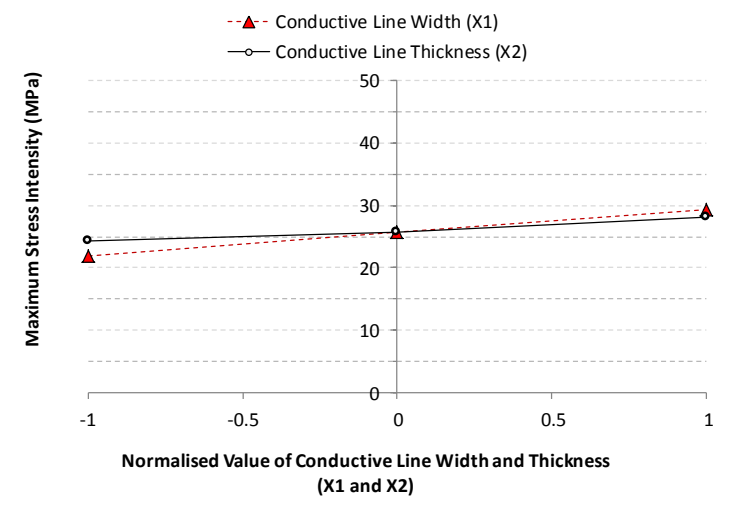

Figure 9: Effect of conductive line width and thickness.

Figure 10 details a set of parametric study results on geometric and material parameters associated with the insulator (see Table 1, parameters X3, X4 and X5). In terms of design parameters, the model predictions show that the insulating material thickness variation has least impact on the observed damage parameter (maximum stress intensity). The largest effect is found with the CTE of the insulating material. These results should be considered taking into account the design range for each parameter over which the stress predictions are obtained and that any other parameters of the printed structure are kept at their nominal values, as detailed in Table 1. 


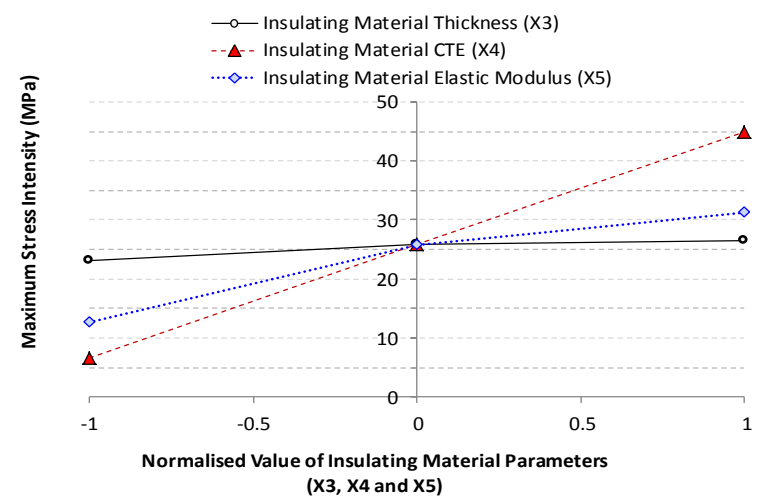

Figure 10: Effect thickness, CTE and elastic modulus of insulating material thickness.

\section{Inkjet Printed Electronics: Design-for-Reliability Modelling Approach}

Changes in the values of the six design parameters detailed in Table 1 result in a range of different designs of the printed structure. The parameters account for the most likely design differences that will be found in the case of this type of printed structure, i.e. the geometric design of the conductive traces, the choice of the insulating ink (and hence physical properties) and the severity of the thermal load condition (being test or use). Understanding how these parameters impact the magnitude of induced thermo-mechanical stress due to CTE differential miss-match between the insulating material and the sintered silver traces can help designers to minimise the risks of delamination damage.

\subsection{Surrogate Modelling}

A model that can predict stress in the printed structure that can then be used to evaluate the behaviour of different design alternatives can be a valuable design tool for assessing what-if scenarios and competing designs. Ultimately, it can be used to minimise the risk of defects and unreliability by designing the structure with the knowledge of what the resulting stresses will be.

An approach based on the integrated use of Designof-Simulations and response surface modelling is adopted in this study to generate a simple surrogate model for the stress in printed silver ink lines in the design space of six parameters detailed in Table 1 . This approach is more beneficial than simple parametric studies as the entire design space can be fully explored and parameter interaction and varying sensitivity in the design space can be explicitly and accurately captured.

Firstly, we employ design-of-experiments methods to identify a set of design points, in this case in the sixdimensional design space of the parameters $X 1$ to $X 6$ $(n=6)$, that are best suited to provide information on effects of these parameters on the conductive line stress response to a thermal load. The Central Composite Design method used here consists of: (1) all factorial design points (i.e. all possible combinations of low and high range values for the six $(n=6)$ parameters, $2 n=64$ points), (2) axial points (i.e. points where one parameter is at low or high range value and all other parameters are at the mid-range value, $2 n=12$ points) and (3) the central point of the design space (i.e. the point at which all parameters are at mid-range values). Thus, for the investigated design structure illustrated in Figure 2, this DoE method results in 77 design configurations. The parametrised finite element model used to derive the results reported in the previous section is used to make predictions for the maximum stress (the damage parameter) in the conductive line for each case.

The FE stress results for all design points are then used to derive a surrogate mode by means of data fitting and in the form of step-wise linear regression:

$$
\begin{aligned}
& \ln \left(\sigma_{I N T}\right)=f(\mathrm{X} 1, \mathrm{X} 2, \mathrm{X} 3, \mathrm{X} 4, \mathrm{X} 5, \mathrm{X} 6)= \\
& a_{0}+\sum_{i=1}^{6} a_{i} \mathrm{X} i+\sum_{i=1}^{6} \sum_{j=1}^{6} b_{i j} \mathrm{X} i \mathrm{X} j
\end{aligned}
$$

The surrogate model detailed in Eq. 2 is in the form of a polynomial approximation that seeks to provide best fit of the set of DoE values for the maximum stress intensity in the printed conductive line obtained from FEA. The model input values, $X 1$ to $X 6$ are the normalised values of the design parameters over the range $[-1,1]$ as defined in Table 1. The model output is the logarithm value of the stress where stress is in the unit of MPa. Table II lists the polynomial coefficients of the derived model. 
The quality of the constructed surrogate model is observed to standard statistical measures used to assess the accuracy of data fitting. In this case it is found that both the $\mathrm{R}$-squared and adjusted $\mathrm{R}$-squared values are 0.999 . The root mean square error is 0.0329 . These measures, along with additional error evaluations and ANOVA [14], indicate extreme accuracy of the data fit achieved with this approximation. Hence, the derived surrogate model can be used not just for qualitative but also for quantitative analysis where the model predictions for maximum stress intensity in the conductive line can be utilised as required instead of using computationally intensive FEA. Figure 11 shows the actual (FEA predicted) versus the approximate (Surrogate model) log-stress values.

\begin{tabular}{|r|c|c|c|}
\hline \multicolumn{2}{|c|}{$\begin{array}{c}\text { Polynomial } \\
\text { Surrogate Model } \\
\text { Term }\end{array}$} & Coefficient & $\begin{array}{c}\text { Coefficient Value } \\
\text { for the Model } \\
\text { Term }\end{array}$ \\
\hline 0 & Constant & a0 & 3.24268408 \\
\hline 1 & X1 & a1 & 0.06586864 \\
\hline 2 & X3 & a3 & 0.10009105 \\
\hline 3 & X4 & a4 & 0.96091091 \\
\hline 4 & X5 & a5 & 0.35187210 \\
\hline 5 & X6 & a6 & 0.80472198 \\
\hline 6 & X1*X2 & b12 & 0.07738479 \\
\hline 7 & X1*X3 & b14 & 0.02494977 \\
\hline 8 & X1*X5 & b15 & 0.07778606 \\
\hline 9 & X2*X5 & b16 & 0.05681907 \\
\hline 10 & X3*X5 & b25 & -0.04833512 \\
\hline 11 & $X 4 * X 4$ & b34 & -0.40424736 \\
\hline 12 & X5*X5 & b36 & -0.25916313 \\
\hline 13 & X6*X6 & b45 & -0.29382596 \\
\hline
\end{tabular}

Table II: Polynomial coefficients of surrogate stress model. Model output is the natural logarithm value of stress, In (stress), where stress is in unit of MPa.

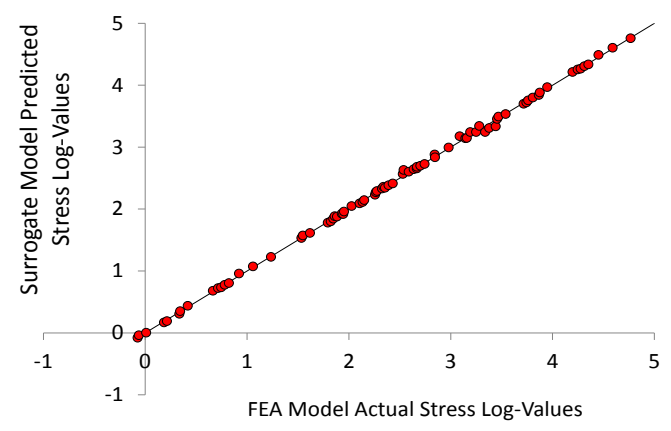

Figure 11: Actual (FEA) versus predicted (Surrogate Model) stress intensity responses

Figure 12 provides two surface plot visual interpretations of the model-predicted stress in twodimensional space of parameter pairs. The other parameters have been set to mid-range values in these plots. It is evident that non-linear behaviour exists, particularly as design and loading condition start to tailor towards high-range values of the parameters.
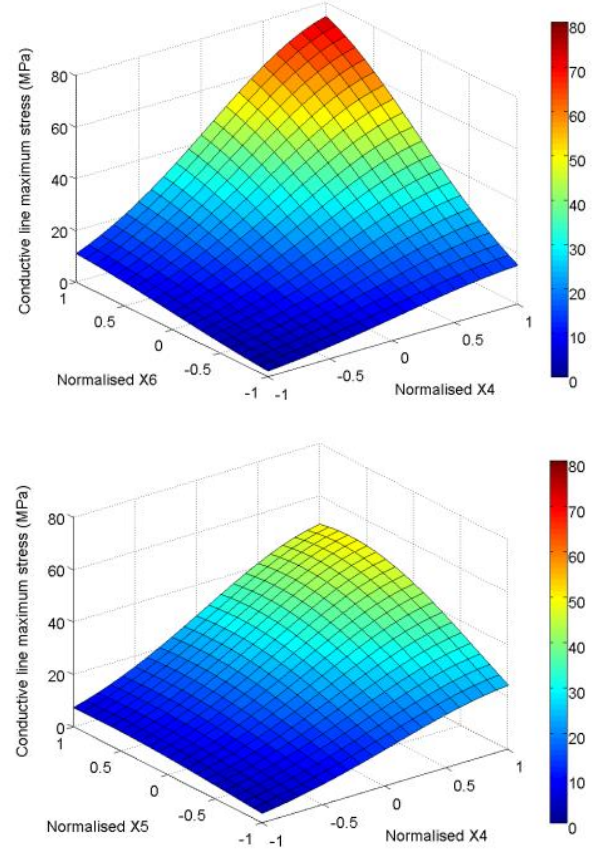

Figure 12: Conductive line maximum stress in normalised space of design parameter pairs: (1) insulator CTE (X4) and applied temperature load (X6) (top) and (2) insulator elastic modulus (X5) and applied temperature load (X6) (bottom). Values of the other four parameters are fixed to the respective mid-value in the parameter range. 


\section{Manufacture and Test}

In order to assess the reliability of the test structure (illustrated in Figure 3), a series of industry-standard validation tests were performed on samples formed using the NextFactory manufacturing process. The NextFactory system based at the Fraunhofer Institute for Manufacturing Engineering and Automation (IPA) was utilised in a process optimisation study so a test rig which mimicked the actual system was used for test sample production.

\subsection{D manufacturing process}

As previously discussed, the NextFactory system is a modular manufacturing system. The approach is based on Photopolymer-Jetting. It consists of different modules that can be changed and modified as needed to guarantee a high degree of flexibility. Material deposition printing modules used Fujifilm Dimatix S-Class and Q-Class printheads. Pinning was carried out using an Ultraviolet (UV) LED (Phoeson FireFly $75 \times 20$, USA) module with a wavelength peak of $395 \mathrm{~nm}$. This module is used to cure the UV ink to a gel-like state so that no more spreading is possible. Conductive ink tracks are exposed to heat radiation from a conventional IR lamp (Efbe-Schott IR 812, Germany) to dry solvents and to prevent unwanted spreading. To ensure accurate thin layers an IPA-designed levelling module formed from a stainless steel roller has been implemented. An alternative/auxiliary UV curing lamp (Excelitas OmniCure S2000, USA) that uses a mercury vapor light source can be used for layer or final curing.

These modules are fixed above a three axis system into a fully automated process chain. Other modules can be readily integrated into the system due to its modular nature. In this case, thermal sintering has been performed. However, the IR and NIR lamps integrated into the NextFactory system could also be used. A pick and place system has been used to place the embedded zero Ohm resistors into the test structure.

The insulating material used for the test structures presented in this paper was developed by TIGER Coatings $\mathrm{GmbH} \& \mathrm{Co}$. KG. The ink is an inkjet printable building material based on acrylates and shows high thermal and chemical stability in its cured state. The ink is still under development and will be adapted according to the needs of the Photopolymer-Jetting manufacturing of microsystems. The conductive tracks were formed using an ink was purchased from Clariant Produkte (Deutschland) $\mathrm{GmbH}$, which sinters thermally at a temperature of $100-200{ }^{\circ} \mathrm{C}$ needing $30-60$ min and can be printed with Fujifilm Dimatix, Inc. S- and Q-Class printheads.

The manufacturing sequence, illustrated in figure 13 utilises different drying modes to process the different material types. The required resolution of the UV curable non-conductive building material is dependent on the wetting behaviour. If the rate of spreading of the ink on the substrate is considered excessive, a pinning process is used to partially cure the material to reduce or eliminate flow. When all passes are printed the height of the deposited layer is adjusted by using the levelling unit, after which the layer will be cured. For the conductive silver ink the process consists of an IR evaporation step to remove solvents form the ink.

Subsequently, the sintering process is performed. When all layers are printed final curing takes place and the sequence ends. For this paper building and conductive material were printed sequentially, but with the proposed modular process chain, this can also be done in parallel. The pick and place, material dispense and inspection modules can be activated at any layer during the build process. This enables the system to be highly flexible and allows integration of a conductive path or any kind of other functional material in a freeformed polymer body. Thus, a high degree of freedom in design and integration of new functionalities is possible. 


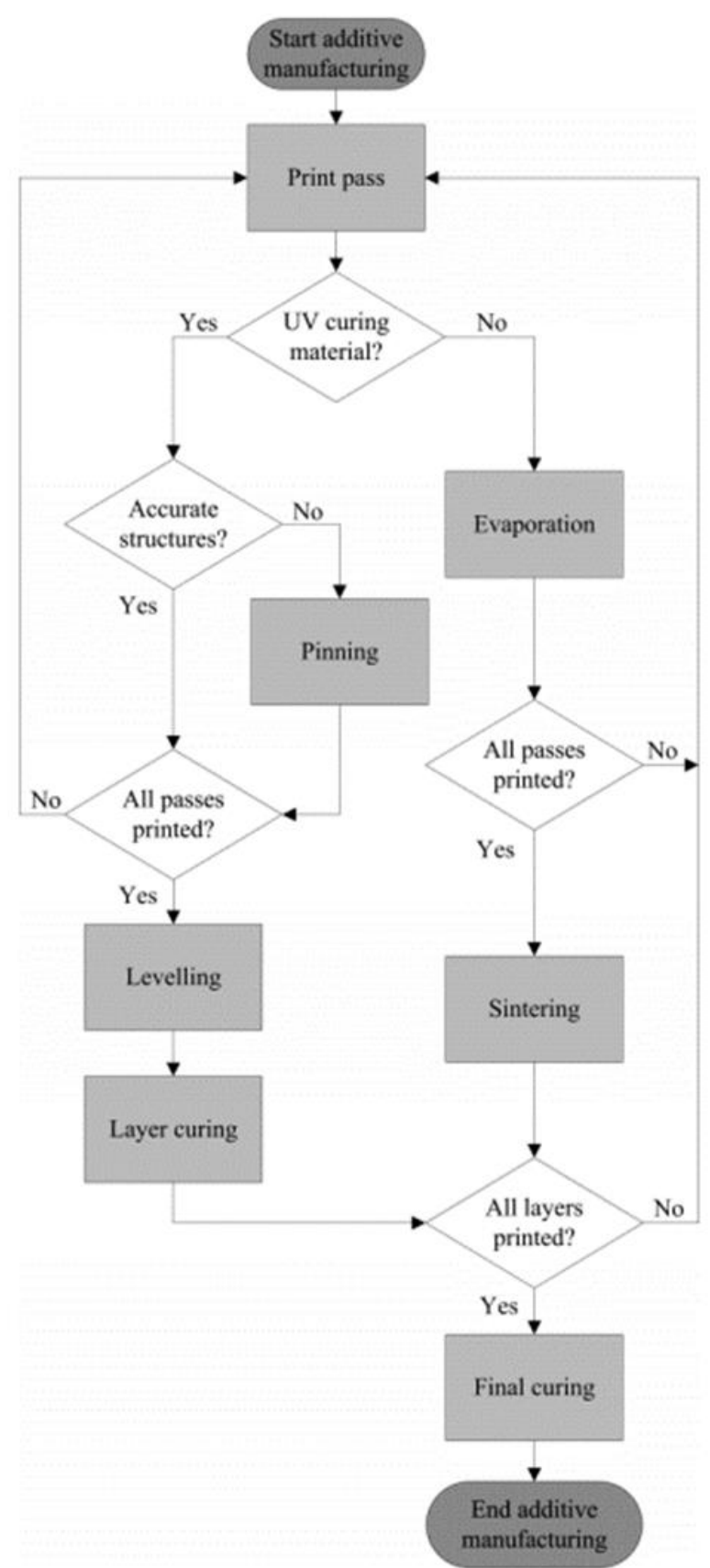

Figure 13: NextFactory manufacturing sequence

\subsection{Reliability testing process}

This manufacturing process was used to form samples for traditional reliability testing. Figure 14 illustrates the final printed part, along with the CAD model it was developed from.
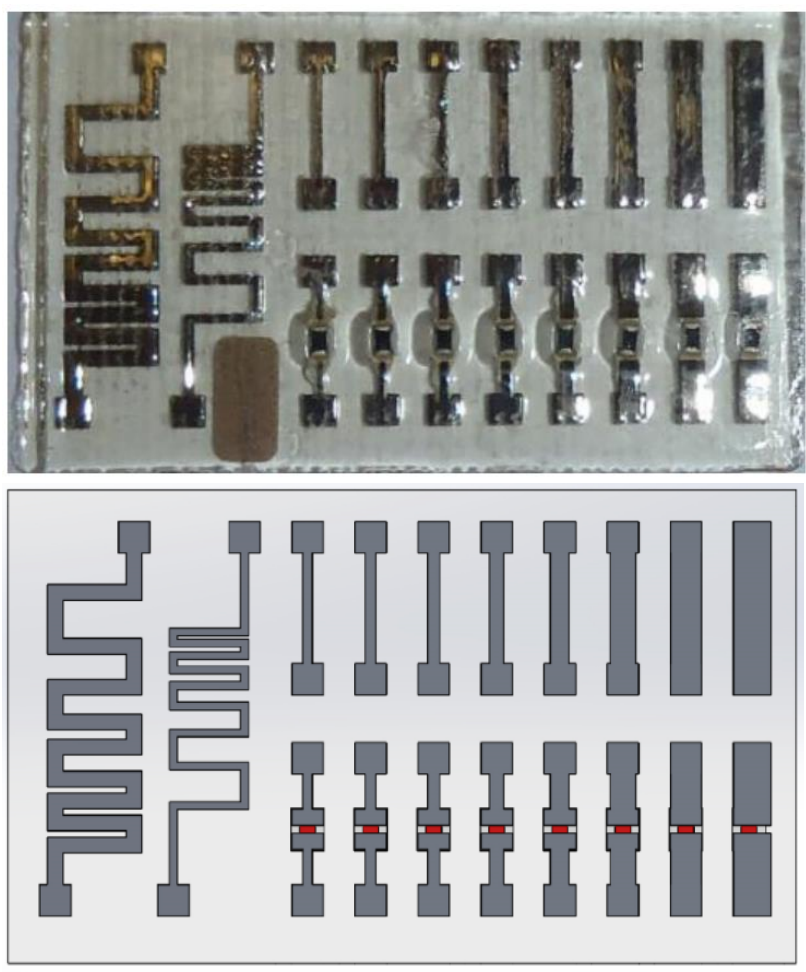

Figure 14: Image of 3D printed test structure and CAD model used as source.

The test samples were subjected to the tests identified in table III. Tests were carried out by Microsemi Corporation (UK). The 3D printed structures passed all tests. While it would be of scientific interest to perform these reliability tests on non-optimal design samples to assess the resulting performance differences, resource limitations preclude such a study. The available resources were instead solely focussed on performing a full reliability study on the optimal designs.

Temperature cycling was performed on 3 prototypes as per JESD22-A104 C [15] using a VOTSCH VT 7012 S2. Initially the intention was to verify that the prototypes could withstand up to 10 cycles as per JESD22-A104 C condition G; when this specification was met MSL decided to test the prototypes to destruction. After 1000 temperature cycles the prototypes were still functional so testing was terminated. 
Hot temperature storage was performed on 3 prototypes as per Mil-Std-883, Method 1008 [16] adhering to test condition B which equated to 24 hours exposure at $125^{\circ} \mathrm{C}$. The equipment used was a Sanyo OMT box oven. No issues were found after hot temperature storage in respect to continuity of functional channels however an increase in warpage was observed and attributed to further curing of the dielectric material during temperature storage.

Cold temperature storage was performed on 3 prototypes as per JED22-A119 [15]; the test condition selected was condition $A$ which equated to a minimum of 168 hours exposure at $-40^{\circ} \mathrm{C}$. The equipment used was a FRIGOR GLE2 0 operating at $-45^{\circ} \mathrm{C}$. Initially the intention was to verify that the prototypes could withstand $168 \mathrm{hrs}$ cold temperature storage. However, when this specification was met it was decided to test the prototypes up to 1000 hours. No issues were found after 1000 hours of cold temperature storage in respect to continuity of functional channels.

Initially it was the intention to perform damp heat as per JESD22-A101-B [15] for 1000 hours with bias, in order to perform the test with bias it was necessary to attach wires to the test pads on the prototype. Unfortunately, this proved not to be viable as attempting to attach the wires resulted in delamination of the 3D printed conductive ink. Several alternate techniques to attach the wires required to deliver the biased voltage/current were attempted but these were also unsuccessful. Therefore after it was decided to perform the damp heat test without bias loading, therefore the test performed was JESD22-A101B 1000 hours at $85^{\circ} \mathrm{C} / 85 \%$ humidity-without bias loading. Analysis of the results clearly shows that the test structures successfully passed the $85 / 85$ validation testing. However, due to significant oxidization of the Silver 3D printed tracks approx. 300-600 Ohms resistance was measured across the channels and the test probe needed to be pressed firmly in order to verify continuity.

MSL testing was performed on 3 prototypes as per JEDEC J-STD [15] ; the test condition selected was Level $2 \mathrm{a}$ which equated to 120 hours pre-conditioning at $60^{\circ} \mathrm{C} / 60 \%$ humidity followed by 3 reflows with $260^{\circ} \mathrm{C}$ peak temperature. The equipment used was a Wewon Model NQ-80-OYO (pre-conditioning) and a Heller 1809 MK III (reflow at $260^{\circ} \mathrm{C}$ peak). Analysis of the MSL 2a validation results shows that the test structures successfully passed the MSL 2a test regime.

Shock and vibration testing was performed on 3 prototypes as per Mil-Std-883 method 2002 and 2007 [16]; the test condition selected was condition B which equated to:

- Mechanical shock- MIL-STD-883 Method 2002, Cond. B. 5 shocks $\times 6$ directions, $1500 \mathrm{~g}$, and $0.5 \mathrm{~ms}$ half sine.

- Mechanical vibration- MIL-STD-883 Method 2007, Cond. A, $20-2000 \mathrm{~Hz}, 20 \mathrm{~g}$.

The equipment used was a Structural Dynamics Systems Shock rig-Mechanical shock and a Ling electronics model SCO-1000-Mechanical vibration system. A test jig was designed and fabricated in order to hold the prototypes during the shock and vibration tests. Analysis of the shock and vibration results shows that the test structures successfully passed the shock and vibration testing.

A chemical resistance test consisting of immersing the prototypes into concentrated fruit juice (orange juice) and storing at $4^{\circ} \mathrm{C}$ for a period of 28 days was performed on 3 prototypes. The results from the chemical resistance test were very encouraging and no visual differences were noted. The validation testing performed clearly shows that the materials and process chain is reliable for single layer packages 


\begin{tabular}{|c|c|c|}
\hline Test & Detail & Result \\
\hline $\begin{array}{l}\text { Temperature } \\
\text { cycling: }\end{array}$ & $\begin{array}{c}+125^{\circ} \mathrm{C} /-40{ }^{\circ} \mathrm{C} 400 \\
\text { cycles [JESD22-A104 C } \\
\text { Cond G] }\end{array}$ & Pass \\
\hline $\begin{array}{l}\text { Hot temperature } \\
\text { storage: }\end{array}$ & $\begin{array}{c}125^{\circ} \mathrm{C} / 24 \text { hrs [Mil-Std- } \\
883 \text {, Method } 1008 \text { Cond } \\
\text { B] }\end{array}$ & Pass \\
\hline $\begin{array}{l}\text { Cold } \\
\text { temperature } \\
\text { storage: }\end{array}$ & $\begin{array}{c}-40{ }^{\circ} \mathrm{C} / 250 \mathrm{hrs}[\mathrm{JESD} 22- \\
\mathrm{A} 1 \mathrm{19}]\end{array}$ & Pass \\
\hline Damp heat: & $1000 \mathrm{hrs}$ at $85^{\circ} \mathrm{C} / 85 \%$ & Pass \\
\hline MSL Testing: & $\begin{array}{l}120 \mathrm{hrs} \text { pre-conditioning } \\
\text { at } 60^{\circ} \mathrm{C} / 60 \% \text { humidity } \\
\text { followed by } 3 \text { reflows with } \\
260^{\circ} \mathrm{C} \text { peak temperature }\end{array}$ & Pass \\
\hline $\begin{array}{l}\text { Shock \& } \\
\text { Vibration }\end{array}$ & $\begin{array}{l}5 \text { shocks in } 6 \text { directions / } \\
1500 \mathrm{~g} / 0.5 \mathrm{~ms} \text { half sine } \\
\text { and } 20-2000 \mathrm{~Hz} / 20 \mathrm{~g}\end{array}$ & Pass \\
\hline $\begin{array}{l}\text { Chemical } \\
\text { resistance }\end{array}$ & 1 month in fruit juice & Pass \\
\hline $\begin{array}{c}\text { High } \\
\text { Temperature }\end{array}$ & $\begin{array}{c}175^{\circ} \mathrm{C} \text { for } 1 \mathrm{hr} \text { [Dielectric } \\
\text { only] }\end{array}$ & Pass \\
\hline $\begin{array}{l}\mathrm{Pb} \text { Reflow } \\
\text { Profile }\end{array}$ & $\begin{array}{c}\left(218^{\circ} \mathrm{C} \text { peak) [Dielectric }\right. \\
\text { only] }\end{array}$ & Pass \\
\hline $\begin{array}{l}\mathrm{Pb} \text { Free Reflow } \\
\text { Profile }\end{array}$ & $\begin{array}{c}\left(245^{\circ} \mathrm{C} \text { peak) [Dielectric }\right. \\
\text { only] }\end{array}$ & Pass \\
\hline
\end{tabular}

Table III: Reliability testing performed on 3D printed test structure

\section{Conclusions}

In this work, a methodology for design, manufacture and testing of 3D printed electronics structures was presented. A representative electronics packaging test structure was assessed using a surrogate modelling approach informed by results from accurate but time consuming Finite Element Analyses. The accuracy of the surrogate model was assessed through $\mathrm{R}$ squared and ANOVA analyses which indicated a very high degree of agreement. Subsequently, a flexible manufacturing process capable of printing the defined test structure was outlined. The system combines inkjet printing and curing of multiple materials, including conductive silver inks, with precision component placement, material dispensing and 3D inspection systems to provide a highly flexible solution for rapid manufacture of electronics packages. The test structures manufactured using the system were subjected to a vigorous set of reliability tests with results indicating that the tests structures met all requirements.

The impact of this study is significant. The capability to manufacture electronics packages using 3D printing processes is becoming increasingly important in the sector. The challenges in design and manufacture, especially in light of the necessarily conservative nature of the sector, are significant. This work demonstrates an effective methodology for evaluating the robustness of the designed product and an effective system for subsequent production. A stringent set of reliability tests were performed on the manufactured products and all were passed. The benefits to the market sector from the proposed methodology are clearly significant.

\section{Acknowledgment}

The authors acknowledge the consortium in the EUfunded project NextFactory (http://www.nextfactoryproject.eu/), and European Community's 7th Framework (FP7/2007-2013) for funding this work under grant agreement No. 608985. 


\section{References}

[1] G. B. Berman, "3-D printing: The new industrial revolution," Business Horizons, vol. 55, no. 2, 2012, pp. 155-162.

[2] Ryan D.Sochol Eric Sweet Casey C.Glick Sung-Yueh Wu Chen Yang Michael Restaino Liwei Lin, 3D printed microfluidics and microelectronics, Microelectronic Engineering, Vol 189, pp. 52-68, April 2018

[3] S. M. Bidoki, D. M. Lewis, M. Clark, A. Vakorov, P. Millner and D. McGorman, "Ink-jet fabrication of electronic components," Journal of Micromechanics and Microengineering," vol. 17, no. 5, 2007, pp. 967974.

[4] J. Perelaer, A. W. M. de Laat, C. Hendriks and U. Schubert, "Inkjet-printed silver tracks: low temperature curing and thermal stability investigation", Journal of Materials Chemistry, vol. 18, 2008, pp. 3209-3215.

[5] 1. Kawahara ,Y., Hodges, S., Cook, B. S., Zhang, C., Abowd, G. D., "Instant inkjet circuits: lab-based inkjet printing to support rapid prototyping of UbiComp devices", Proc UbiComp'13, September 8-12, 2013 , Zurich, Switzerland, pp. 363-372

[6] B. Khorramdel, J. Liljeholm, M. Laurila, T. Lammi, G. Mårtensson, T Ebefors, F. Niklaus, M.Mäntysalo, Inkjet printing technology for increasing the $1 / 0$ density of 3D TSV interposers, Microsystems and Nanoengineering, Vol. 3, 10.04.2017, p. 17002.

[7] http://www.nano-di.com [Accessed: 5 March 2018].
[8] M O'Reilly and Jeff Leal, Jetting your way to fine pitch 3D interconnects, Chip Scale Review, Sept/Oct 2010

[9] NextFactory, http://www.nextfactory-project.eu

[10] S. Stoyanov, C. Bailey, Machine learning for additive manufacturing of electronics, 40th International Spring Seminar on Electronics Technology (ISSE), 2017

[11] G. Tourloukis, S. Stoyanov, T. Tilford, C. Bailey, Predictive modelling for 3D inkjet printing processes, 39 th International Spring Seminar on Electronics Technology (ISSE), 2016, pp. 257-262

[12] B. Hu (2010) "Inkjet Printing of Silver for Direct Write Applications", PhD Thesis, University of Manchester, UK.

[13] Vasiljevic D.Z., Menicanin A.B., Zivanov L.D. Mechanical Characterization of Ink-Jet Printed Ag Samples on Different Substrates. In: CamarinhaMatos L.M., Tomic S., Graça P. (eds) Technological Innovation for the Internet of Things. DoCEIS 2013. IFIP Advances in Information and Communication Technology, vol 394. Springer, Berlin, Heidelberg, 2013

[14] Statistical principles in experimental design, BJ Winer, DR Brown, KM Michels - 1971 - McGraw-Hill New York

[15] www.jedec.org [Accessed: 5 March 2018]

[16] MIL-STD-883E Test Method standard Microcirciuts, US Department of Defense, FSC 5962 , 1996 